Research Article

\title{
Compensatory Analysis and Optimization for MADM for Heterogeneous Wireless Network Selection
}

\author{
Jian Zhou ${ }^{1,2}$ and Can-yan Zhu' \\ ${ }^{1}$ Institute of Intelligent Structure and System, Soochow University, Soochow 215006, China \\ ${ }^{2}$ Department of Information Engineering, Suzhou Global Institute of Software Technology, Soochow 215163, China \\ Correspondence should be addressed to Can-yan Zhu; qiwuzhu@suda.edu.cn
}

Received 3 February 2016; Accepted 5 May 2016

Academic Editor: Rajesh Khanna

Copyright ( 2016 J. Zhou and C.-y. Zhu. This is an open access article distributed under the Creative Commons Attribution License, which permits unrestricted use, distribution, and reproduction in any medium, provided the original work is properly cited.

In the next-generation heterogeneous wireless networks, a mobile terminal with a multi-interface may have network access from different service providers using various technologies. In spite of this heterogeneity, seamless intersystem mobility is a mandatory requirement. One of the major challenges for seamless mobility is the creation of a network selection scheme, which is for users that select an optimal network with best comprehensive performance between different types of networks. However, the optimal network may be not the most reasonable one due to compensation of MADM (Multiple Attribute Decision Making), and the network is called pseudo-optimal network. This paper conducts a performance evaluation of a number of widely used MADMbased methods for network selection that aim to keep the mobile users always best connected anywhere and anytime, where subjective weight and objective weight are all considered. The performance analysis shows that the selection scheme based on MEW (weighted multiplicative method) and combination weight can better avoid accessing pseudo-optimal network for balancing network load and reducing ping-pong effect in comparison with three other MADM solutions.

\section{Introduction}

With the emerging and development of all kinds of wireless access technology, including 2G, 3G, WLAN, WiMax (World Interoperability for Microwave Access), and MBWA (Mobile Broadband Wireless Access), wireless networks overlap and complement each other, forming a hybrid wireless network called heterogeneous wireless networks [1]. To support seamless mobility while a mobile station roams within a heterogeneous wireless network, VHO (Vertical Handoff) necessity estimation and decision to select a best target network are two important aspects of the overall mobility framework. The handoff necessity estimation is important in order to keep the unnecessary handoffs and their failures at a low level. On the other hand, to maximize the endusers' satisfaction level, the decision to select the best network among other available candidates plays an important role as well. The network selection process consists of three major subservices: (1) network monitoring monitors the current network conditions (network availability, signal strength, current call connection, etc.) and provides the data gathered together with information related to the user preferences, current running applications on the user's mobile device, and their QoS requirements; (2) handover decision handles the network selection process (which ranks the candidate networks and selects the best target) and is initiated either by an automatic trigger for handover for an existing call connection or by a request for a new connection on the mobile device; and (3) handover execution: once a new target network is selected, the connection is set up on the target candidate network (and the old connection torn down).

Network selection algorithm has become a more complex problem and combines multiple systems' attributes to choose the target network that offers the highest overall performance. This approach is considered optimal as compared to the other traditional approaches that rely on a single system's attributes like RSS (Received Signal Strength) or available bandwidth to make handoff decisions. As all of these parameters present different ranges and units of measurements, they need to be normalized in order to make them comparable. 
Utility functions are used for normalization to map all the parameters into dimensionless units within the range $[0,1]$ [2-7]. This normalized information is then used in the decision making process in order to compute a ranked list of the best available network choices. MADM including SAW, MEW, GRA, and TOPSIS is widely used as score function methods for network selection [8-15]. User or network operator preferences for the main trade-off criteria can be represented by the use of different weights in weighted score functions. The candidate network with the highest score is selected as the target network if that differs from the current network connection (or it is for a new connection); it prompts handover execution (or new network connection setup).

However, the optimal network with best comprehensive performance may not be the most reasonable one due to compensation of MADM; we call the network pseudo-optimal network. For example, if network with best comprehensive performance and heavy load is selected, which may further aggravate network congestion, end-user cannot enjoy good network quality. Moreover, it is argued that an appropriate MADM should not make end-user access pseudo-optimal network in [10]. Hence, performance of accessing pseudooptimal network is firstly analyzed for SAW (Simple Additive Weighting Method), TOPSIS (Technique for Order Preference by Similarity to Ideal Solution), GRA (Grey Relational Analysis), and MEW, then network selection based on MEW and combinational weight is proposed. It can be seen from simulation that the proposed algorithm can make end-user better avoid assessing pseudo-optimal network and has better performance in network load balance and reducing ping-ping effect.

\section{Compensatory Analysis for MADM}

2.1. Common MADM. MADM algorithms can be divided into compensatory and noncompensatory ones. Noncompensatory algorithms are used to find acceptable alternatives which satisfy the minimum cutoff. On the contrary, compensatory algorithms combine multiple attributes to find the best alternative. Most MADM algorithms that have been studied for the network selection problem are compensatory algorithms.

SAW is widely used by most studies of the network selection problem using cost or utility functions, generally given by

$$
U_{\mathrm{SAW}}=\sum_{j=1}^{n} w_{j} u_{i j},
$$

where $w_{j}$ represents the weight of attribute $c_{j}$ and $u_{i j}$ represents the adjusted value of attribute $c_{j}$ of the network $r_{i}$.

$\mathrm{MEW}$ is to calculate the coefficient by multiplicative operation, given by

$$
U_{\mathrm{MEW}}=\prod_{j=1}^{n} u_{i j}{ }^{w_{j}} .
$$

Other two MADM algorithms used for network selection are TOPSIS and GRA, which both consider the distance from
TABLE 1: Network selection based on SAW.

\begin{tabular}{lccc}
\hline Utility & $w_{j}$ & Network A & Network B \\
\hline$U(C)$ & $1 / 3$ & 0.5 & 0.8 \\
$U(E)$ & $1 / 3$ & 0.5 & 0.8 \\
$U(L)$ & $1 / 3$ & 0.5 & 0.05 \\
Aggregate utility & & 0.5 & 0.55 \\
\hline
\end{tabular}

the evaluated network to one or multiple reference networks. Coefficient of TOPSIS can be calculated as

$$
U_{\text {TOPSIS }}=\frac{D^{\alpha}}{D^{\alpha}+D^{\beta}},
$$

where $D^{\alpha}$ and $D^{\beta}$ represent the Euclidean distances from the current network to the worst and best reference networks, respectively, given by

$$
\begin{aligned}
& D^{\alpha}=\sqrt{\sum_{j=1}^{n} w_{j}^{2}\left(u_{i j}-v_{j}^{\alpha}\right)^{2}}, \\
& D^{\beta}=\sqrt{\sum_{j=1}^{n} w_{j}^{2}\left(u_{i j}-v_{j}^{\beta}\right)^{2}},
\end{aligned}
$$

where $v_{j}^{\alpha}$ and $v_{j}^{\beta}$ represent the values of attribute $c_{j}$ of the worst and best reference networks, respectively.

Different from TOPSIS, GRA uses only the best reference network to calculate the coefficient, given by

$$
U_{\mathrm{GRA}}=\frac{1}{\sum_{j=1}^{n} w_{j}\left|u_{i j}-v_{j}^{\beta}\right|+1} .
$$

2.2. Compensatory Analysis. According to the principle of network selection, the optimal network is the network with best comprehensive performance, but it may be not the most reasonable one due to compensation of MADM. For example, the result is the fact that end-user chooses best network from networks A and B by SAW as shown in Table 1, where decision attributes are cost $(C)$, power consumption $(E)$, and load $(L)$. Assume that only subjective weight is considered.

Table 1 shows that the comprehensive performance of network B is superior to network A, and end-user will choose network $B$ as access network. However, load utility of network $\mathrm{B}$ in Table 1 is 0.05 which is close to 0 ; namely, network $B$ is not suitable for new access due to its heavy load, and otherwise it may lead to congestion for network B and not balance load between networks A and B. So network A should be the reasonable choice for the above situation. But enduser chooses network B due to performance compensation between attributes, which means the excellent performance of cost and power consumption compensates the bad performance of load in network $\mathrm{B}$, and we call the network like network B pseudo-optimal network.

The results that end-user chooses best network from networks A and B by TOPSIS and GRA are shown in Tables 2 and 3 , respectively. It can be seen from Tables 2 and 3 that 
TABLE 2: Network selection based on TOPSIS.

\begin{tabular}{lccc}
\hline Utility & $w_{j}$ & Network A & Network B \\
\hline$U(C)$ & $1 / 3$ & 0.5 & 0.8 \\
$U(E)$ & $1 / 3$ & 0.5 & 0.8 \\
$U(L)$ & $1 / 3$ & 0.5 & 0.05 \\
Aggregate utility & & 0.5 & 0.5333 \\
\hline
\end{tabular}

TABLE 3: Network selection based on GRA.

\begin{tabular}{lccc}
\hline Utility & $w_{j}$ & Network A & Network B \\
\hline$U(C)$ & $1 / 3$ & 0.5 & 0.8 \\
$U(E)$ & $1 / 3$ & 0.5 & 0.8 \\
$U(L)$ & $1 / 3$ & 0.5 & 0.05 \\
Aggregate utility & & 0.6667 & 0.6897 \\
\hline
\end{tabular}

TABLE 4: Network selection based on MEW $\left(w_{l}=1 / 3\right)$.

\begin{tabular}{lccc}
\hline Utility & $w_{j}$ & Network A & Network B \\
\hline$U(C)$ & $1 / 3$ & 0.5 & 0.8 \\
$U(E)$ & $1 / 3$ & 0.5 & 0.8 \\
$U(L)$ & $1 / 3$ & 0.5 & 0.05 \\
Aggregate utility & & 0.5 & 0.3175 \\
\hline
\end{tabular}

TABLE 5: Network selection based on MEW $\left(w_{l}=0.05\right)$.

\begin{tabular}{lccc}
\hline Utility & $w_{j}$ & Network A & Network B \\
\hline$U(C)$ & 0.5 & 0.5 & 0.8 \\
$U(E)$ & 0.45 & 0.5 & 0.8 \\
$U(L)$ & 0.05 & 0.5 & 0.05 \\
Aggregate utility & & 0.5 & 0.6964 \\
\hline
\end{tabular}

TOPSIS and GRA also choose network B with heavy load like SAW, and they have the same limitation.

The result that end-user chooses best network from networks A and B by MEW is shown in Table 4. It can be seen from Table 4, unlike SAW, TOPSIS, and GRA, that enduser chooses network A with light load as access network. However, if we readjust attribute weight in Table 4 and reestimate the comprehensive performance of networks $\mathrm{A}$ and $B$ by MEW, as shown in Table 5 , it can be seen from Table 5 that the comprehensive performance of network B is superior to network A again; end-user will choose network B as access network. Obviously, MEW cannot ensure that enduser can always avoid accessing the pseudo-optimal network.

The reason that end-user chooses optimal network from network A to network B when attribute weight of load is from one-third to 0.05 is shown in Figure 1. In Figure 1, the expression of two curves is $0.5^{w}$ and $0.05^{w}$, respectively, difference of utility value between two curves is larger when $w$ is larger, and difference is close to 0 when $w$ is smaller; hence, the comprehensive performance of network $\mathrm{B}$ will be reduced well due to multiplicative features of MEM and network A is selected as optimal network when difference of load utility between networks A and B is larger because weight of load is one-third. However, network B is selected as optimal network again when difference of load utility between networks $A$ and

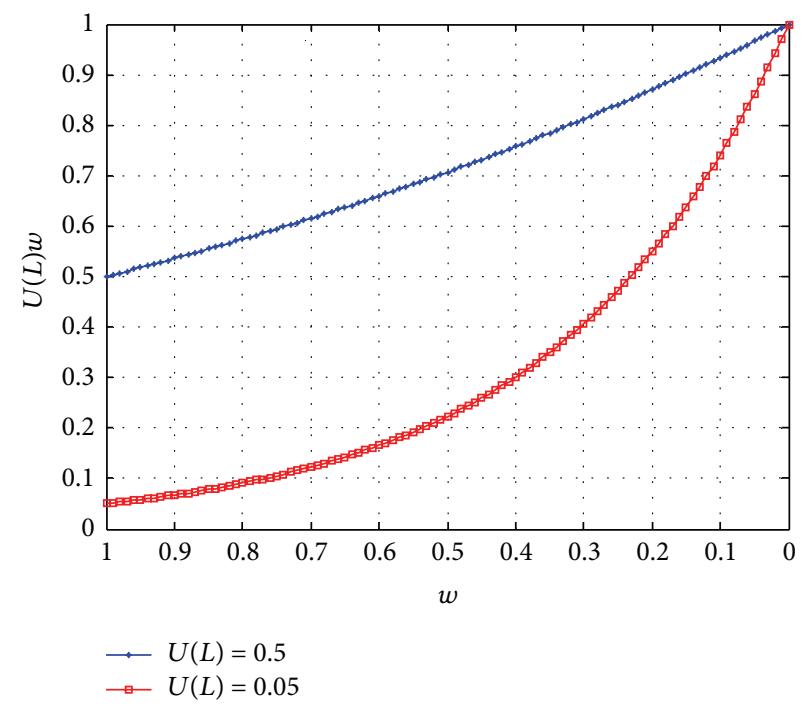

FIgURE 1: Performance comparison of load with different weight.

B is smaller because weight of load is 0.05 , and network A with light load is not selected. Through the above analysis, to make end-user avoid accessing the network with attribute with poor performance, weight of the attribute should be adjusted in real-time to prevent its value from being too small, and MEW should be used as decision making method to rank alternative network.

\section{Network Selection Based on MEW and Combination Weight}

As described in Section 2, attribute weight should be adjusted in real-time to make end-user avoid accessing the pseudooptimal network, while objective weight method can calculate attribute weight according to attribute value of alternative network and recalculate attribute weight when attribute data changes. However, subjective weight must also be considered to reflect experience and subjective importance for attribute of decision makers. Hence, combination weight which integrates the subjective weight and objective weight will be considered. The steps for calculating combination weight are as follows.

Step 1. Objective weight is determined by entropy weighting method, calculated as follows:

(1) Construct normalized matrix R, given by

$$
\mathbf{R}=\left[\begin{array}{ccccc}
u_{11} & \cdots & u_{1 j} & \cdots & u_{1 n} \\
\vdots & & \vdots & \cdots & \vdots \\
u_{i 1} & \cdots & u_{i j} & \cdots & u_{i n} \\
\vdots & & \vdots & \cdots & \vdots \\
u_{m 1} & \cdots & u_{m j} & \cdots & u_{m n}
\end{array}\right]
$$



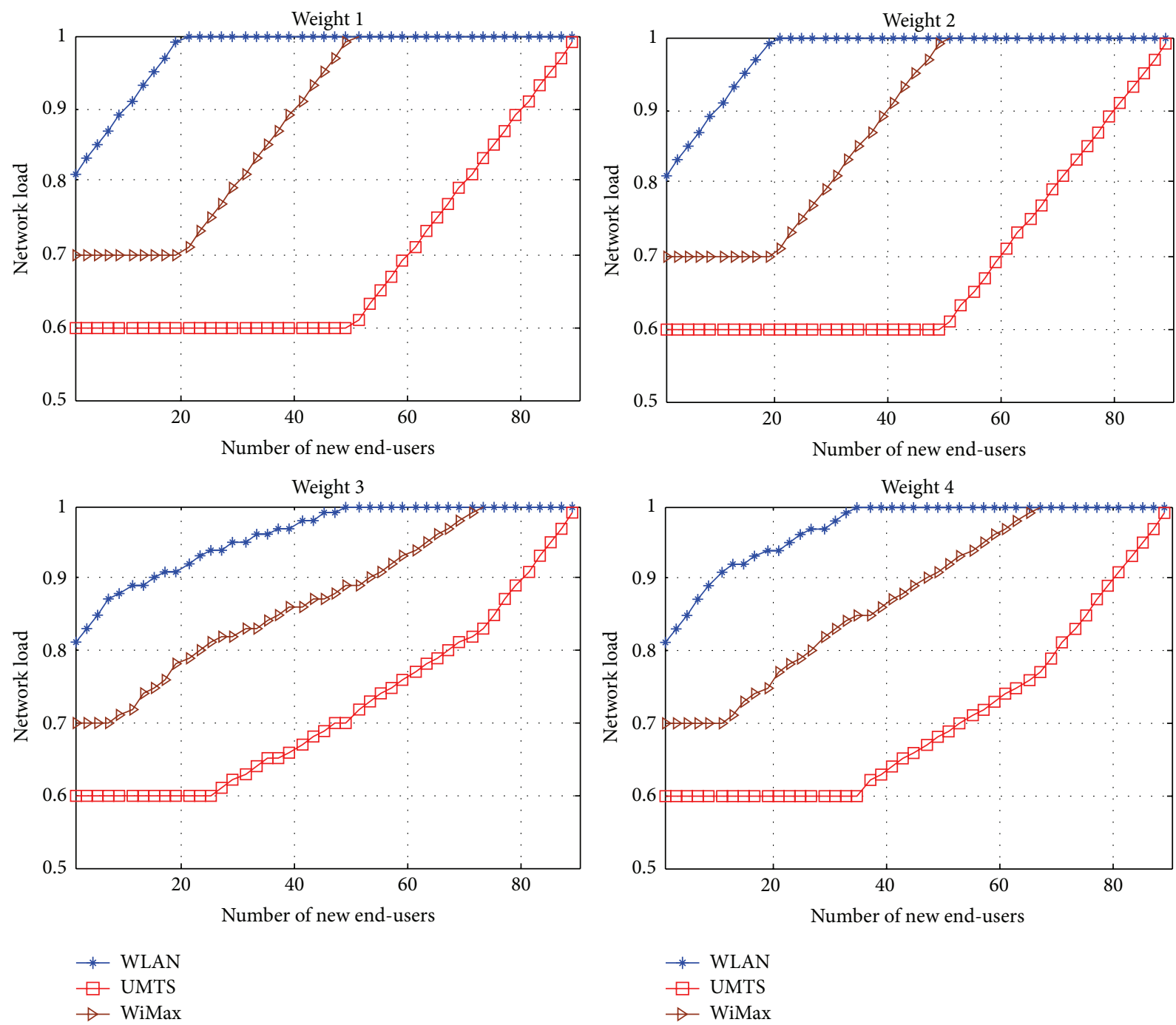

FIGURE 2: Change of network load by SAW.

(2) Calculate information entropy of attribute $c_{j}$, given by

$$
E_{j}=\frac{-1}{\ln m} \sum_{i=1}^{m} u_{i j} \ln u_{i j} \text {. }
$$

(3) Calculate objective weight of attribute $c_{j}$, given by

$$
w_{o j}=\frac{1-E_{j}}{\sum_{k=1}^{n}\left(1-E_{k}\right)} .
$$

Step 2. Subjective weight is determined by experience and assigned directly in this paper, denoted by $w_{s j}$.

Step 3. For considering subjective weight and objective weight, combination weight can be expressed as

$$
w_{c j}=\alpha w_{o j}+\beta w_{s j},
$$

where $\alpha$ and $\beta$ meet $\alpha+\beta=1$ and $\alpha, \beta \geq 0$.
Considering that weighted attribute values determined by subjective weight and objective weight should be consistent, optimal mathematical model can be constructed to solve $\alpha$ and $\beta$. Deviation degree of subjective and objective evaluation value of alternative networks is given by

$$
d_{i}=\sum_{j=1}^{n}\left(\alpha u_{i j} w_{o j}-\beta u_{i j} w_{s j}\right)^{2}
$$

The smaller the value of $d_{i}$ is, the more consistent the subjective and objective evaluations tend to be. Hence, optimal mathematical model can be constructed as follows:

$$
\begin{array}{ll}
\min & \sum_{i=1}^{m} d_{i}=\sum_{i=1}^{m} \sum_{j=1}^{n}\left(\alpha u_{i j} w_{s j}-\beta u_{i j} w_{o j}\right)^{2} \\
\text { s.t. } & \alpha+\beta=1 \\
& \alpha, \beta \geq 0
\end{array}
$$



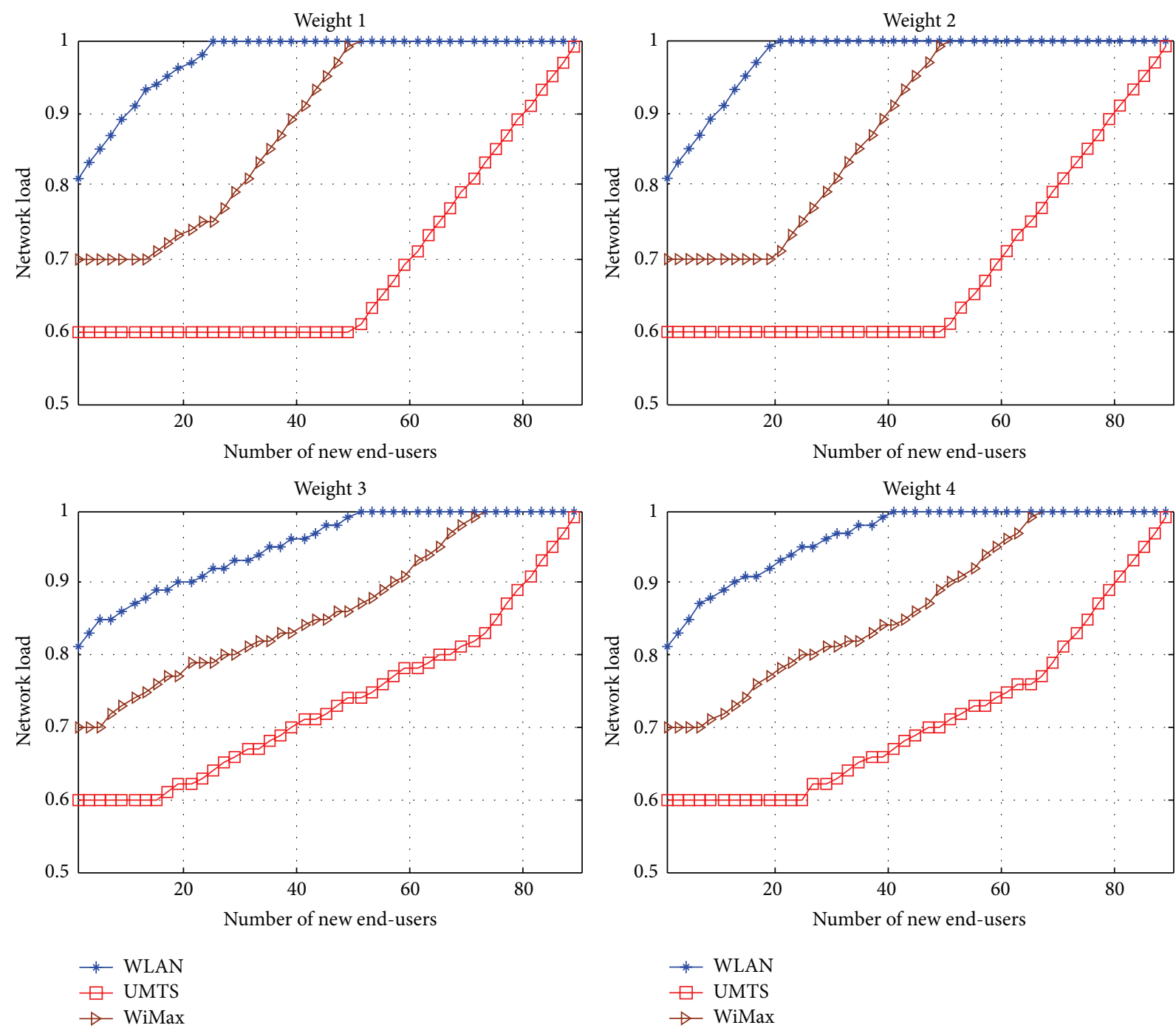

FIgURE 3: Change of network load by TOPSIS.

and its solution can be obtained as

$$
\begin{gathered}
\alpha=\frac{\sum_{i=1}^{m} \sum_{j=1}^{n} u_{i j}^{2} w_{o j}\left(w_{s j}+w_{o j}\right)}{\sum_{i=1}^{m} \sum_{j=1}^{n} u_{i j}^{2}\left(w_{s j}+w_{o j}\right)^{2}} \\
\beta=\frac{\sum_{i=1}^{m} \sum_{j=1}^{n} u_{i j}^{2} w_{s j}\left(w_{s j}+w_{o j}\right)}{\sum_{i=1}^{m} \sum_{j=1}^{n} u_{i j}^{2}\left(w_{s j}+w_{o j}\right)^{2}} .
\end{gathered}
$$

Substitute (13) into (10); combination weight can be determined.

Step 4. Substitute $w_{c j}$ into (2); rank for alternative networks can be obtained by MEW.

\section{Simulation and Analysis}

In this section, two groups of simulations are used to validate performance of the proposed algorithm; one simulation is for performance evaluation of network load balance and the
TABLE 6: Measurement value of attribute and parameter setting for utility function.

\begin{tabular}{lccccc}
\hline Attribute & WLAN & UMTS & WiMax & $x_{\alpha}$ & $x_{\beta}$ \\
\hline$C($ cent $/ \mathrm{Mb})$ & 10 & 50 & 30 & 0 & 100 \\
$E(\mathrm{w})$ & 2 & 6 & 3 & 0 & 10 \\
$L(\%)$ & 80 & 60 & 70 & 0 & 100 \\
$D(\mathrm{kbps})$ & 220 & 400 & 350 & 200 & 800 \\
\hline
\end{tabular}

Notes: $x_{\alpha}$ and $x_{\beta}$ are lower limit and upper limit of linear utility function.

other is for performance evaluation of reducing ping-pong effect.

In simulation environment, three networks WLAN, UMTS, and WiMax are selected as alternative networks, and cost, power consumption, load, and data rate (B) are decision attribute used for wireless network selection. Linear utility function is adopted as utility function for all attributes. Measurement value of attribute and parameter setting for utility function is shown in Table 6, and measurement 

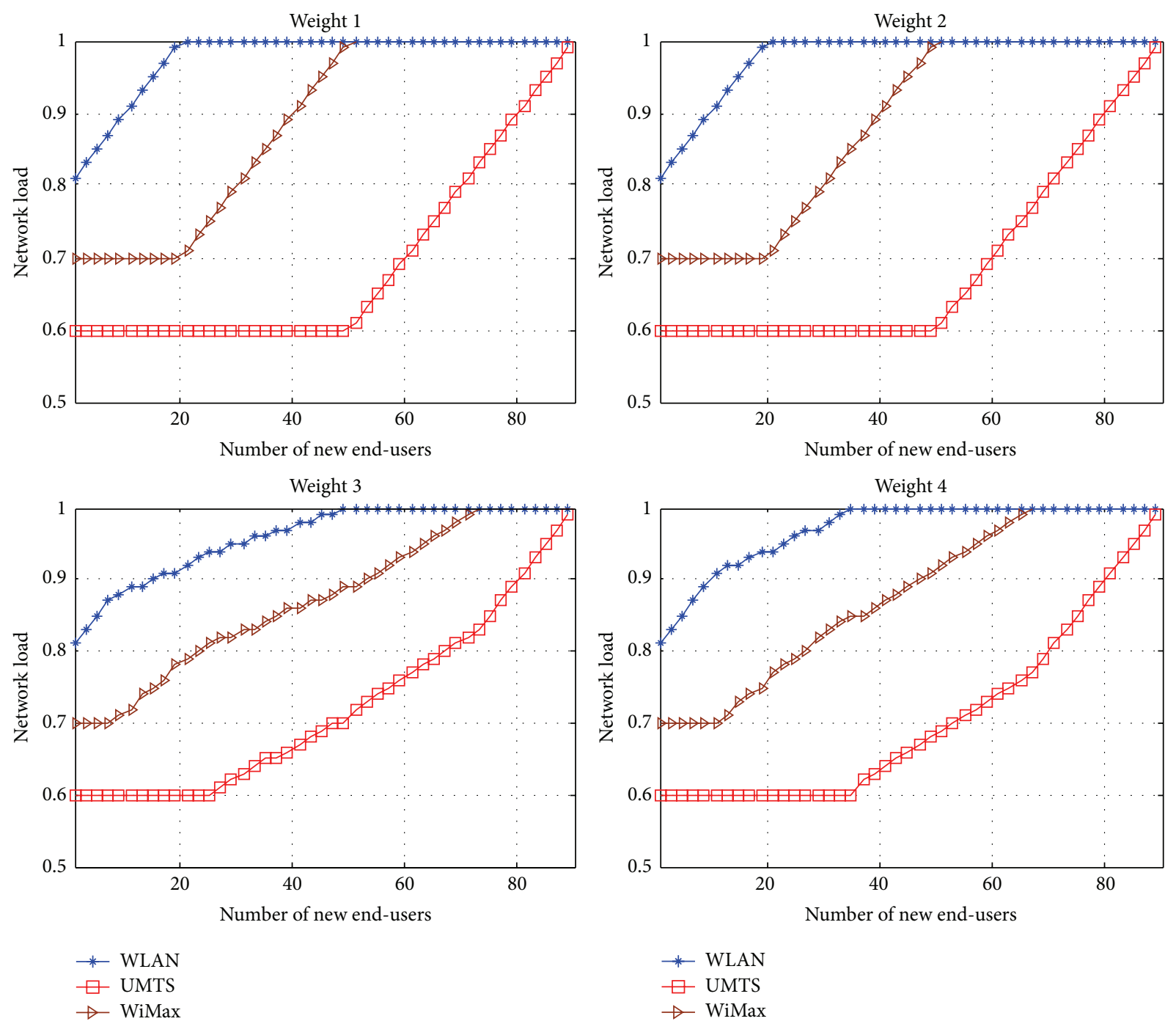

FIGURE 4: Change of network load by GRA.

TABLE 7: Utility used for load balance simulation.

\begin{tabular}{lccc}
\hline Utility & WALN & UMTS & WiMax \\
\hline$U(C)$ & 0.9 & 0.5 & 0.7 \\
$U(E)$ & 0.8 & 0.4 & 0.7 \\
$U(L)$ & 0.2 & 0.4 & 0.3 \\
\hline
\end{tabular}

values of load and data rate will change after beginning of simulation.

4.1. Simulation for Performance of Network Load Balance. According to Table 6, price, power consumption, and load are selected as decision attributes; utility and weight of decision attributes are shown in Tables 7 and 8, respectively.

Assume that there are new requests coming constantly in overlapping area of WLAN, UMTS, and WiMax, and enduser accesses the optimal network based on network selection algorithm. Then, Figures 2-5 show change of network load
TABLE 8: Weight for network attributes.

\begin{tabular}{ll}
\hline Weight & $(C, E, L)$ \\
\hline Weight 1 & Only subjective weight $(1 / 3,1 / 3,1 / 3)$ \\
Weight 2 & Only subjective weight $(0.5,0.45,0.05)$ \\
& Combination of subjective weight $(1 / 3$, \\
& $1 / 3,1 / 3)$ and objective weight \\
Weight 3 & computed by $(9)$ \\
& Combination of subjective weight $(0.5$, \\
& $\begin{array}{l}\text { C.45, } 0.05) \text { and objective weight } \\
\text { Computed by }(9)\end{array}$ \\
\hline
\end{tabular}

of alternative network when end-user selects optimal network by SAW, TOPSIS, GRA, and MEW, respectively, where attribute weight is in case of weight 1 , weight 2 , weight 3 , and weight 4 , respectively. In Figures $2-5$, there are a number of new end-users on horizontal coordinates and network load on vertical coordinates. 

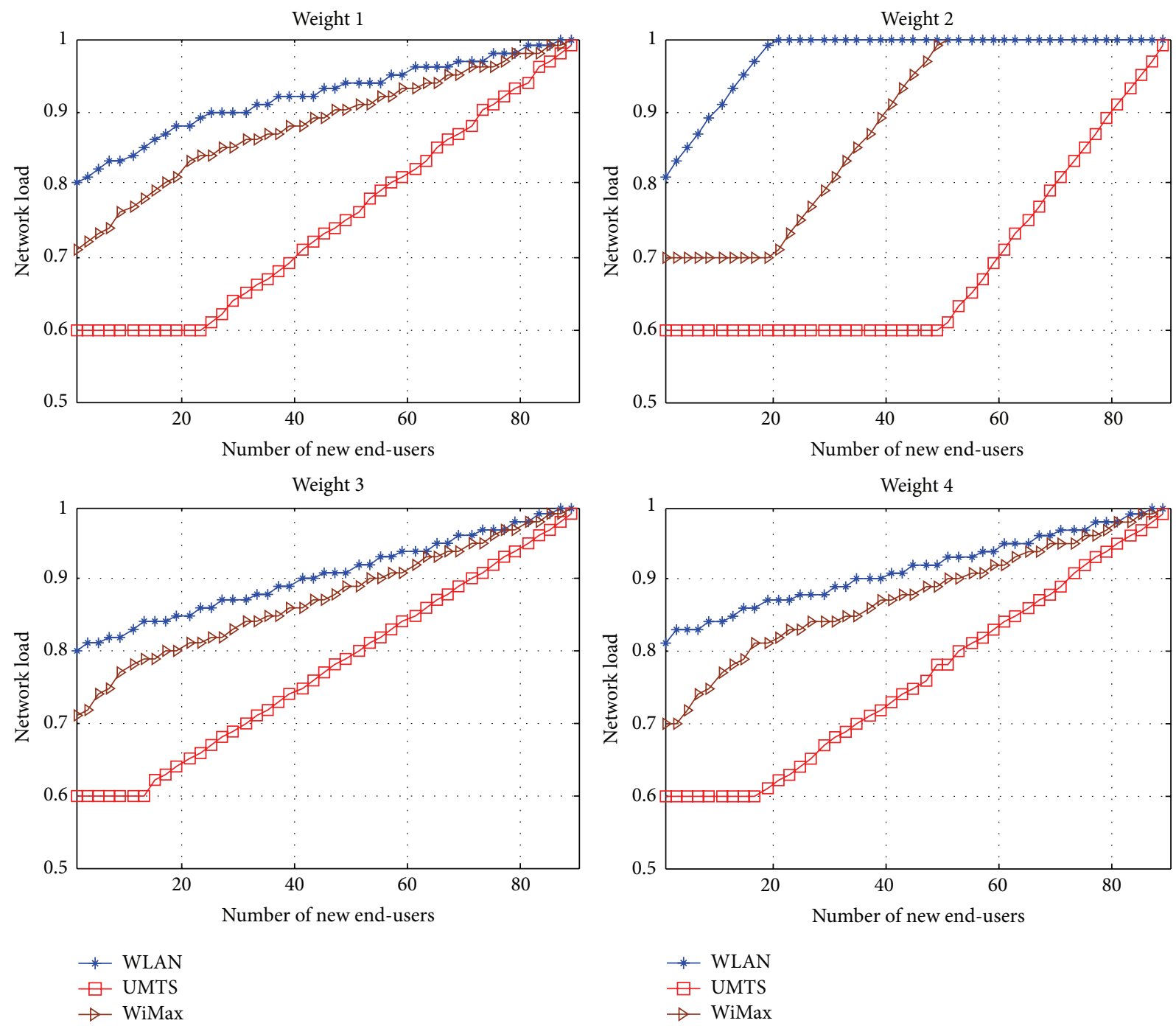

FIGURE 5: Change of network load by MEW.

Figure 2 shows change of network load when optimal network is selected by SAW. The order of optimal network selected by new end-users is much the same for four weight cases. It is failed to balance network load well, and it is easy to cause congestion for network with heavy load so it cannot provide good quality for end-user.

Changes of network load when optimal network is selected by TOPSIS and GRA, respectively, are shown in Figures 3 and 4, where the performance is like SAW.

Figure 5 shows change of network load when optimal network is selected by MEW. It can be seen from Figure 5 that network load can be balanced better by MEW when attribute weight is in case of weight 1 , weight 3 , and weight 4. Hence, no matter what value of subject weight is, end-user can avoid better accessing of the pseudo-optimal network and balance network load between three alternative networks by the scheme based on MEW and combination weight.

4.2. Simulation for Performance of Ping-Pong Effect. According to Table 6, price, power consumption, and data rate are
TABLE 9: Utility used for ping-pong effect simulation.

\begin{tabular}{lccc}
\hline Utility & WALN & UMTS & WiMax \\
\hline$U(C)$ & 0.9 & 0.5 & 0.7 \\
$U(E)$ & 0.8 & 0.4 & 0.7 \\
$U(B)$ & 0.0625 & 0.125 & 0.25 \\
\hline
\end{tabular}

selected as decision attributes; utility of decision attributes is shown in Table 9 and weight of decision attributes is as in Table 8.

Assume that data rate will vary by around $50 \mathrm{kbps}$ after start of simulation. Figures 6-9 show performance of pingpong effect of alternative network when end-user selects optimal network by SAW, TOPSIS, GRA, and MEW, respectively, where attribute weight is in case of weight 1 , weight 2 , weight 3 , and weight 4, respectively. In Figures 6-9, there are a number of data rate fluctuations on horizontal coordinates and a number of network handoffs on vertical coordinates. 


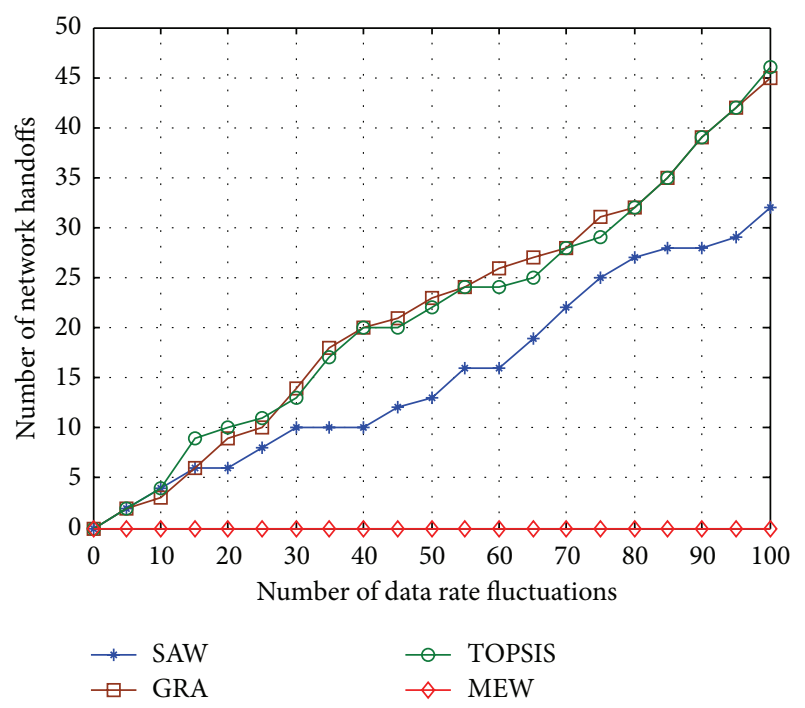

FIGURE 6: Network handoffs in case of weight 1.

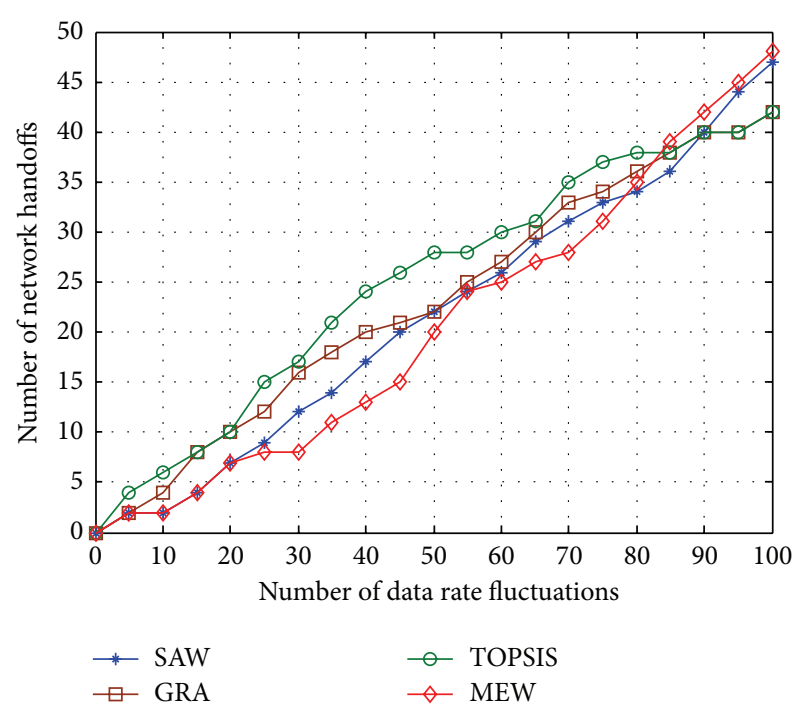

Figure 7: Network handoffs in case of weight 2.

It can be seen from Figures 6-9 that network handoff is more frequent when optimal network is selected by SAW, TOPSIS, and GRA, because data rate of WLAN may be lower than the minimum requirements of end-user for data rate fluctuation, and end-user must select other eligible networks. However, there are fewer network handoffs when optimal network selected by MEW is in case of weight 1, weight 3, and weight 4 . Hence, no matter what value of subject weight is, end-user can avoid better accessing of the pseudo-optimal network and reduce network handoffs between alternative networks by the scheme based on MEW and combination weight, which is a better solution for ping-pong effect caused by data rate fluctuations.

4.3. Analysis and Comparison of Algorithm Complexity. In this section, network selection algorithm based on SAW,

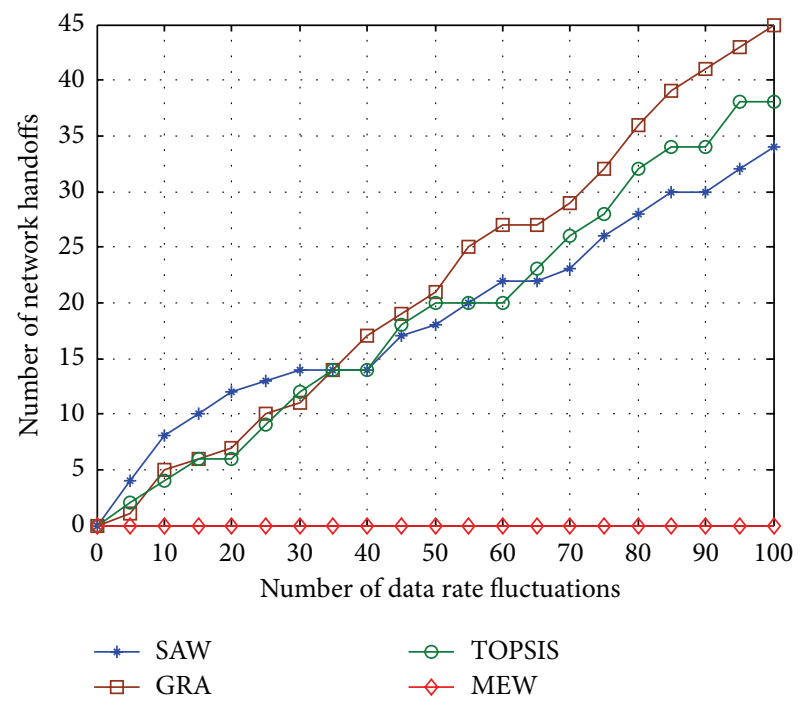

FIGURE 8: Network handoffs in case of weight 3.

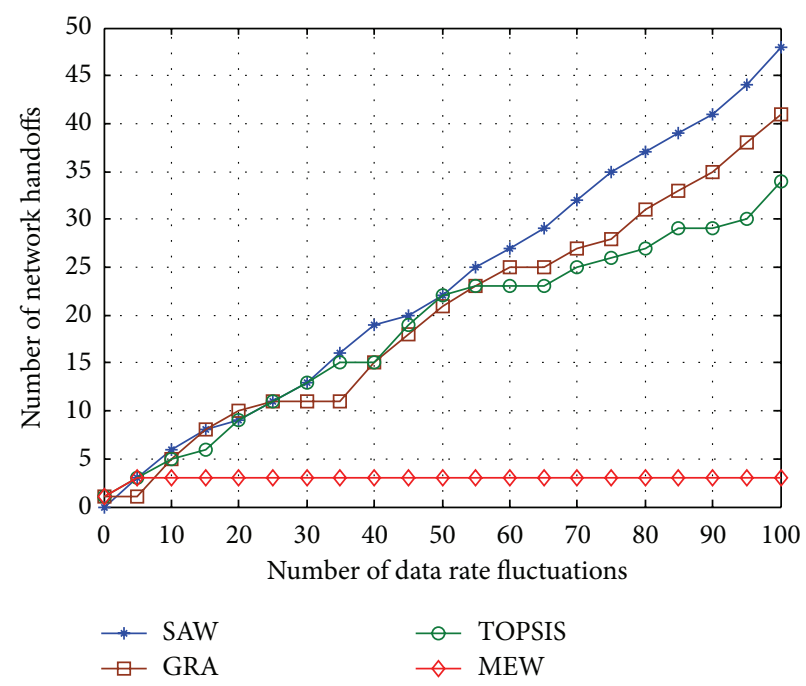

Figure 9: Network handoffs in case of weight 4.

TABLE 10: Comparison of computational overhead.

\begin{tabular}{lcccc}
\hline & SAW & TOPSIS & GRA & MEW \\
\hline Weight 3 & 1.471 us & 1.562 us & 1.521 us & 1.575 us \\
Weight 4 & 1.470 us & 1.560 us & 1.522 us & 1.578 us \\
\hline
\end{tabular}

TOPSIS, GRA, and MEW is implemented on DSP device, respectively, and computational overhead of network selection process is computed. In Table 10, mean computational overhead of one network selection process is showed when three networks are alternative and three decision attributes are chosen.

It can be seen from Table 10 that computational overhead of MEW is about $7 \%, 0.8 \%$, and $3 \%$ more than SAW, TOPSIS, and GRA, respectively, but the performance of balancing network load and reducing ping-pong effect of MEW is vastly better than the others. 


\section{Conclusions}

In this paper, network selection algorithm based on MEW and combination weight is proposed for the problem that pseudo-optimal network may be chosen because of compensation of attribute performance when optimal network is selected by SAW, TOPSIS, and GRA. Simulation shows that the proposed algorithm not only makes end-user avoid accessing pseudo-optimal network, but also balances network load to prevent network congestion and reduce pingpong effect, which improves system performance.

\section{Competing Interests}

The authors declare that they have no competing interests.

\section{Acknowledgments}

This work is financially supported by NSFC no. 61071214 .

\section{References}

[1] M. Corici, J. Fiedler, T. Magedanz, and D. Vingarzan, "Access network discovery and selection in the future wireless communication," Mobile Networks and Applications, vol. 16, no. 3, pp. 337-349, 2011.

[2] Q.-T. Nguyen-Vuong, G. D. Yacine, and N. Agoulmine, "On utility models for access network selection in wireless heterogeneous networks," in Proceedings of the IEEE Network Operations and Management Symposium (NOMS '08), pp. 44-151, Salvador, Brazil, April 2008.

[3] I. Chamodrakas and D. Martakos, "A utility-based fuzzy TOPSIS method for energy efficient network selection in heterogeneous wireless networks," Applied Soft Computing Journal, vol. 12, no. 7, pp. 1929-1938, 2012.

[4] L. Wang and G.-S. G. S. Kuo, "Mathematical modeling for network selection in heterogeneous wireless networks-a tutorial," IEEE Communications Surveys and Tutorials, vol. 15, no. 1, pp. 271-292, 2013.

[5] R. Trestian, O. Ormond, and G.-M. Muntean, "Enhanced power-friendly access network selection strategy for multimedia delivery over heterogeneous wireless networks," IEEE Transactions on Broadcasting, vol. 60, no. 1, pp. 85-101, 2014.

[6] P. Kosmides, A. Rouskas, and M. Anagnostou, "Utility-based RAT selection optimization in heterogeneous wireless networks," Pervasive and Mobile Computing, vol. 12, no. 6, pp. 92111, 2014.

[7] R. Chai, H. Zhang, X. Dong, Q. Chen, and T. Svensson, "Optimal joint utility based load balancing algorithm for heterogeneous wireless networks," Wireless Networks, vol. 20, no. 6, pp. 1557-1571, 2014.

[8] J. Liu and X.-N. Li, "Handover algorithm for WLAN/cellular networks with analytic hierarchy process," Journal on Communications, vol. 34, no. 2, pp. 65-72, 2013.

[9] S. Zhang and Q. Zhu, "Heterogeneous wireless network selection algorithm based on group decision," Journal of China Universities of Posts and Telecommunications, vol. 21, no. 3, pp. $1-9,2014$.

[10] Q.-T. Nguyen-Vuong, N. Agoulmine, E. H. Cherkaoui, and L. Toni, "Multicriteria optimization of access selection to improve the quality of experience in heterogeneous wireless access networks," IEEE Transactions on Vehicular Technology, vol. 62, no. 4, pp. 1785-1800, 2013.

[11] R. Trestian, O. Ormond, and G.-M. Muntean, "Performance evaluation of MADM-based methods for network selection in a multimedia wireless environment," Wireless Networks, vol. 21, no. 5, pp. 1745-1763, 2014.

[12] L. Xu and Y. Li, "A network selection scheme based on topsis in heterogeneous network environment," Journal of Harbin Institute of Technology, vol. 21, no. 1, pp. 43-48, 2014.

[13] M. Drissi and M. Oumsis, "Performance evaluation of multicriteria vertical handover for heterogeneous wireless networks," in Proceedings of the 1st International Conference on Intelligent Systems and Computer Vision (ISCV '15), pp. 1-5, IEEE, Fez, Morocco, March 2015.

[14] R. Verma and N. P. Singh, "GRA based network selection in heterogeneous wireless networks," Wireless Personal Communications, vol. 72, no. 2, pp. 1437-1452, 2013.

[15] N. P. Singh and B. Singh, "Vertical handoff decision in 4G wireless networks using multi attribute decision making approach," Wireless Networks, vol. 20, no. 5, pp. 1203-1211, 2014. 


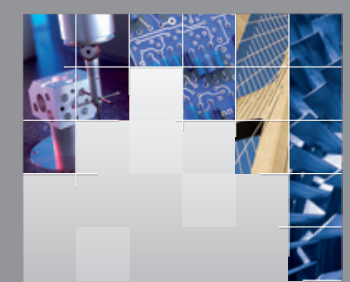

\section{Enfincering}
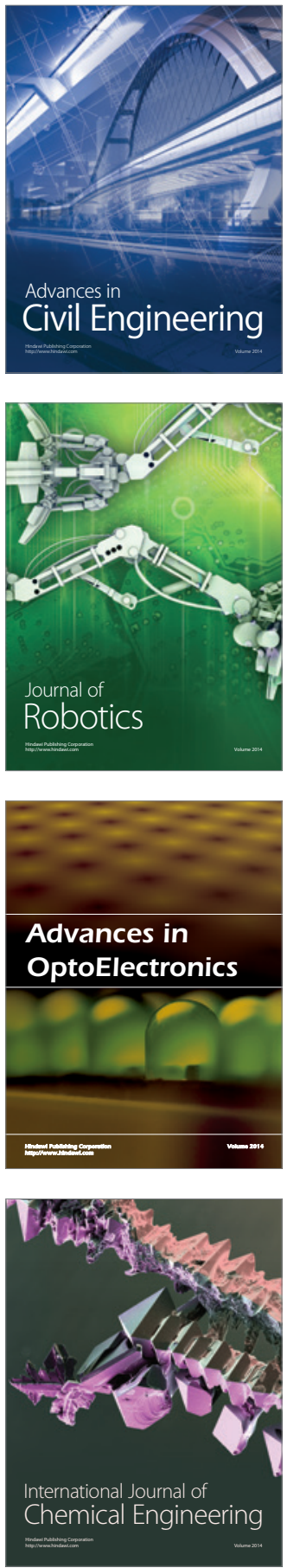

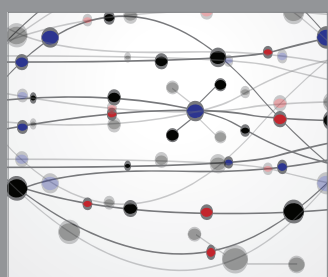

The Scientific World Journal

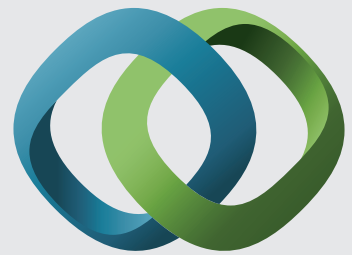

\section{Hindawi}

Submit your manuscripts at

http://www.hindawi.com
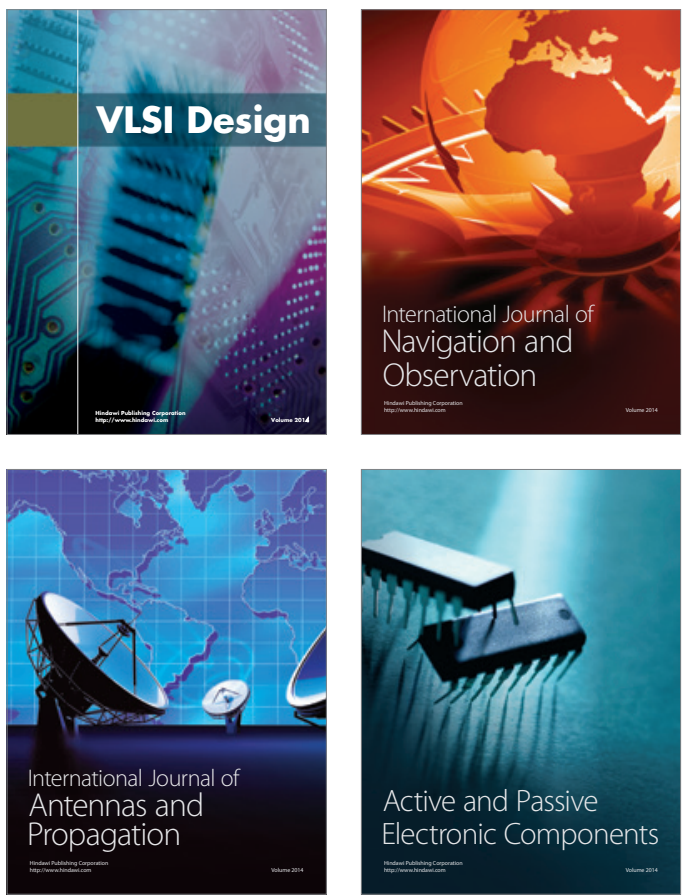
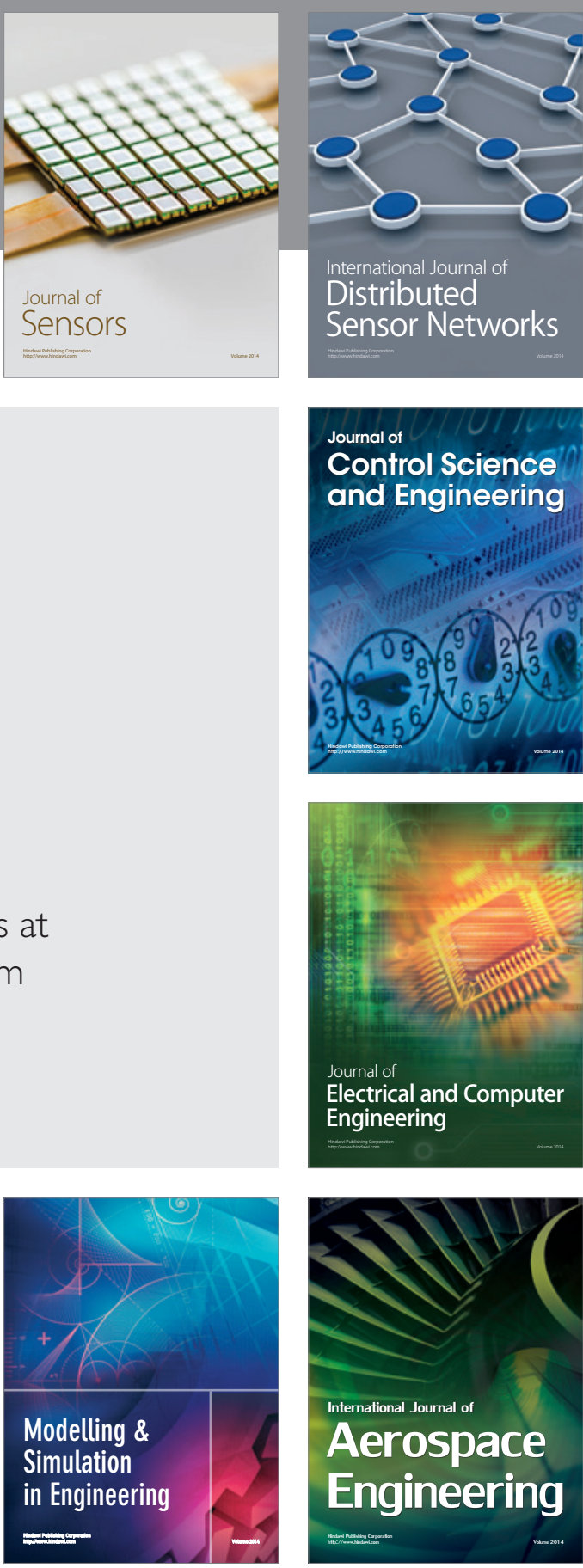

International Journal of

Distributed

Sensor Networks

Journal of

Control Science

and Engineering
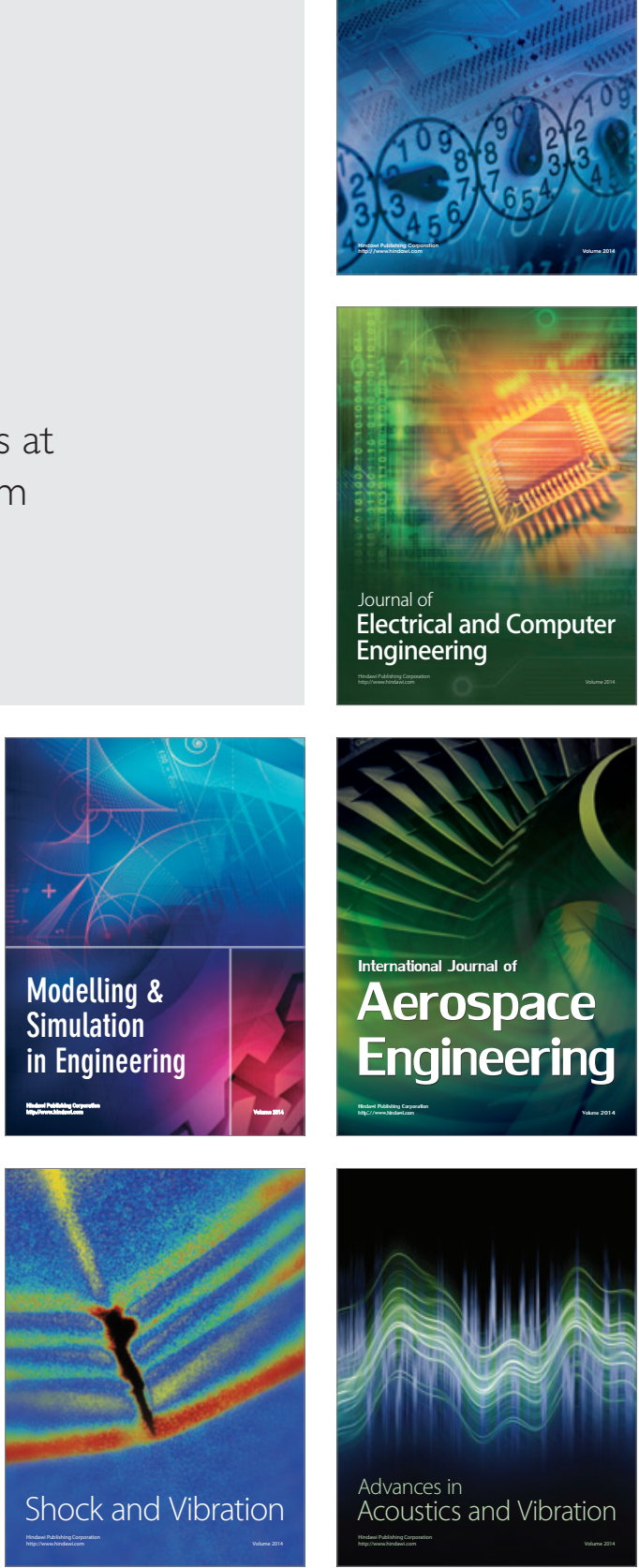